\title{
Interwoven Four-Compartment Capillary Membrane Technology for Three-Dimensional Perfusion with Decentralized Mass Exchange to Scale Up Embryonic Stem Cell Culture
}

\author{
Jörg C. Gerlach ${ }^{\mathrm{a}}$ Marc Lübberstedt ${ }^{c}$ Josefina Edsbagge ${ }^{\mathrm{e}}$ Alexander Ring ${ }^{\mathrm{c}}$ Mariah Hout $^{\mathrm{a}}$ \\ Matt Baun $^{a} \quad$ Ingrid Rossberg $^{c}$ Fanny Knöspel ${ }^{c} \quad$ Grant Peters $^{a} \quad$ Klaus Eckert $^{d}$ \\ Annika Wulf-Goldenberg ${ }^{d}$ Petter Björquist ${ }^{\mathrm{e}}$ Harald Stachelscheid $^{c}$ Thomas Urbaniak $^{c}$ \\ Gerald Schatten $^{\mathrm{b}}$ Toshio Miki ${ }^{\mathrm{a}}$ Eva Schmelzer ${ }^{\mathrm{a}}$ Katrin Zeilinger ${ }^{c}$ \\ ${ }^{a}$ Departments of Surgery and Bioengineering, McGowan Institute for Regenerative Medicine, and \\ bPittsburgh Development Center, University of Pittsburgh, Pittsburgh, Pa., USA; ' AG Experimentelle Chirurgie \\ Campus Virchow Clinic, Charité - Universitätsmedizin Berlin, and ${ }^{\mathrm{d}}$ Max Delbrück Center for Molecular Medicine,

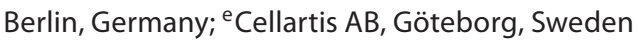

\section{Key Words}

Embryonic stem cells • Three-dimensional perfusion •

Expansion • Bioreactors

\begin{abstract}
We describe hollow fiber-based three-dimensional (3D) dynamic perfusion bioreactor technology for embryonic stem cells (ESC) which is scalable for laboratory and potentially clinical translation applications. We added 2 more compartments to the typical 2-compartment devices, namely an additional media capillary compartment for countercurrent 'arteriovenous' flow and an oxygenation capillary compartment. Each capillary membrane compartment can be perfused independently. Interweaving the 3 capillary systems to form repetitive units allows bioreactor scalability by multiplying the capillary units and provides decentralized media
\end{abstract}

The bioreactor prototypes were produced by Stem Cell Systems $\mathrm{GmbH}$ (Berlin, Germany), a spin-off of the Charité, Berlin. They were purchased. J.C.G. licensed intellectual property to the company. perfusion while enhancing mass exchange and reducing gradient distances from decimeters to more physiologic lengths of $<1 \mathrm{~mm}$. The exterior of the resulting membrane network, the cell compartment, is used as a physically active scaffold for cell aggregation; adjusting intercapillary distances enables control of the size of cell aggregates. To demonstrate the technology, mouse ESC (mESC) were cultured in 8- or 800-ml cell compartment bioreactors. We were able to confirm the hypothesis that this bioreactor enables $\mathrm{mESC}$ expansion qualitatively comparable to that obtained with

\section{Abbreviations used in this paper}

2D two-dimensional

3D three-dimensional

ESC embryonic stem cells

FACS fluorescence-activated cell sorting

HE hematoxylin-eosin

MEF mouse embryonic fibroblasts

mESC mouse embryonic stem cells

PBS phosphate-buffered saline

SSEA-1 stage-specific embryonic antigen-1

\section{KARGER}

(๑) 2010 S. Karger AG, Basel

Fax +4161306 1234

E-Mail karger@karger.ch

www.karger.com
Accessible online at: www.karger.com/cto
Dr. Jörg C. Gerlach

Departments of Surgery and Bioengineering

McGowan Institute for Regenerative Medicine, University of Pittsburgh

3025 East Carson Street, Pittsburgh, PA 15203 (USA)

Tel. +1 412383 7460, Fax +1 412383 9460, E-Mail joerg.gerlach@ cellnet.org 
Petri dishes, but on a larger scale. To test this, we compared the growth of 129/SVEV mESC in static two-dimensional Petri dishes with that in 3D perfusion bioreactors. We then tested the feasibility of scaling up the culture. In an $800-\mathrm{ml}$ prototype, we cultured approximately $5 \times 10^{9}$ cells, replacing up to 800 conventional $100-\mathrm{mm}$ Petri dishes. Teratoma formation studies in mice confirmed protein expression and gene expression results with regard to maintaining 'stemness' markers during cell expansion.

Copyright $\odot 2010$ S. Karger AG, Basel

\section{Introduction}

Because of their unique characteristics, embryonic stem cells (ESC), initially generated from mice [Evans and Kaufman, 1981; Martin, 1981] and later from humans [Thomson et al., 1998], hold great potential as a cell source for applications in basic science, pharmacological drug screening, toxicity testing and cell-based therapies in regenerative medicine.

Scale-up of ESC production, e.g. for potential clinical translation research, aims to achieve ESC amounts on the order of $10^{9}$ or higher, preferably in the same closed system that can be used for follow-up differentiation. Current Petri dish and flask techniques represent two-dimensional (2D) culture methods and provide static open systems with discontinuous medium exchange; they are labor intensive and impractical for handling larger cell numbers.

Hollow-fiber capillary membrane bioreactor technology enables dynamic and continuous perfusion culture conditions, increased cell density to physiological levels [Cukierman et al., 2002; Abbott, 2003; Powell, 2005] and scale-up of cell production [Gerlach et al., 2003; Monga et al., 2005]. However, the typical 2-compartment bioreactor - a cell compartment surrounding a bundle of hydrophilic porous capillaries with external oxygenation and nutrition mainly via diffusion - is limited [Xu and Reid, 1999] by nonuniform mass exchange over decimeters of capillary length.

We describe a hollow fiber-based three-dimensional (3D) dynamic perfusion bioreactor technology for ESC which is scalable for laboratory and potentially clinical translation applications, offering a single system for both cell expansion and follow-up differentiation and satisfying closed-system requirements. We added a second hydrophilic compartment for countercurrent 'arteriovenous' medium flow and a third hydrophobic compartment for oxygenation and $\mathrm{CO}_{2} / \mathrm{pH}$ regulation (fig. 1) to the typical 2-compartment devices. To overcome the limitations of typical devices, the 3 capillary sets were also interwoven into repetitive units. The exterior of the resulting membrane network, representing the cell compartment, thus provides a physically active scaffold for cell aggregation. Interweaving the 4 compartments into repetitive units enables bioreactor scalability by simply multiplying the capillary units and provides decentralized medium perfusion while enhancing mass exchange and reducing gradient distances from decimeters to more physiologic lengths of $<1 \mathrm{~mm}$. This bioreactor technology platform provides a closed system and a culture environment suitable for good manufacturing practice, facilitating biotechnological applications and potentially clinical translation of ESC research.

We hypothesized that this bioreactor would enable mESC expansion qualitatively comparable to that obtained with Petri dishes, but on a larger scale. To test this, we compared the growth of $129 / \mathrm{SVEV}$ mESC in static 2D Petri dishes with that in $3 \mathrm{D}$ perfusion bioreactors. We then tested the feasibility of scaling up the culture from an 8 -ml cell compartment to an $800-\mathrm{ml}$ prototype. In the latter, we cultured approximately $5 \times 10^{9}$ cells, replacing up to 800 conventional $100-\mathrm{mm}$ Petri dishes.

\section{Materials and Methods}

\section{Bioreactor and Perfusion Periphery}

The multicompartment bioreactors are composed of 3 independent yet interwoven hollow-fiber capillary membrane systems (fig. 1A; compartments II-IV) that are integrated into a 2-component polyurethane potting/housing [725A (isocyanate)/725B (polyol), Room \& Haas, Bremen, Germany]. Two of the hydrophilic medium-perfusion capillary membranes are made of microporous polyethersulfone with a molecular weight cut-off of approximately MW 500,000 (Membrana, Wuppertal, Germany; internal diameter $300 \pm 40 \mu \mathrm{m}$, wall thickness $100 \pm 25 \mu \mathrm{m}$, pore size $0.5 \pm 0.1 \mu \mathrm{m})$. The third is made of hydrophobic multilaminate hollow-fiber membrane capillaries (MHF, Mitsubishi, Tokyo, Japan; internal diameter $200 \pm 10 \mu \mathrm{m}$, wall thickness $42 \pm$ $3 \mu \mathrm{m}, \mathrm{O}_{2}$ permeability $>0.8 \times 10^{5} \mathrm{~cm}^{3} / \mathrm{cm}^{2} / \mathrm{s} / \mathrm{cm} \mathrm{Hg}$ ) to enable gas exchange. Thus, the cells located within the extracapillary space (fig. 1A; cell compartment, I) are exposed to decentralized medium and plasma supply with high mass exchange rates and direct membrane oxygenation via flow-enhanced diffusion. For cell injection, a flow head connected to open-ended silicone rubber capillaries (Silastic, Dow Corning, New York, N.Y., USA) is used. All materials used are medical grade and already in clinical use in blood oxygenators or plasma filters, which would enable a fast transition into potential clinical translation applications.

The bioreactor is integrated into a processor-controlled perfusion device with electronic pressure and flow regulation. Modular pump units with exchangeable multichannel flow heads and gears 
serve for medium recirculation and substitution. The modular pumps allow the use of one $800-\mathrm{ml}$ cell compartment bioreactor or up to four $8-\mathrm{ml}$ bioreactors in parallel (fig. 1D). A heating unit provides a constant temperature within the perfusion circuit. Flow rates of air, $\mathrm{O}_{2}, \mathrm{CO}_{2}$ and $\mathrm{N}_{2}$ are controlled by a gas mixing unit. For further details, see the legend to figure 1 . The perfusion tubing with bubble traps was made of standard medical-grade dialysis polyvinyl chloride (B. Braun, Melsungen, Germany). Sterilization was performed with formaldehyde gas at $60^{\circ} \mathrm{C}$ with alternating degassing.

Preparation of Cells

129/SVEV mESC from passage 11 (CMTI-1, Chemicon International, Billerica, Mass., USA) were cocultured with the mitomycin-inactivated mouse embryonic fibroblast (MEF) strain CD1 (passage 5), isolated according to protocols from the WiCell Research Institute (Madison, Wisc., USA). Mouse ESC were seeded at a cell density of $3.5 \times 10^{4}$ cells $/ \mathrm{cm}^{2}$ into culture dishes coated with $0.1 \%$ gelatine (EmbryoMax ES Cell Qualified, Millipore, Bedford, Mass., USA) and preseeded with inactivated MEF seeded at a density of $3.0 \times 10^{4}$ cells $/ \mathrm{cm}^{2}$. Cells were cultured in T175 culture flasks (BD Falcon, San José, Calif., USA) or in Luminox multiwell plates suitable for immune fluorescence studies (TC Quality, Bio-One, Greiner). Every 2-3 days, cells were passaged enzymatically, using a $0.05 \%$ trypsin- $0.02 \%$ EDTA solution (Biochrom, Berlin, Germany).

Day 0 cultures represent 2D mESC/MEF cocultures run in parallel to those used for bioreactor cultivation (studied on the day of inoculation).

Fluorescence-activated cell sorting (FACS) and immunoreactivity studies indicated that the inoculated cell population already exhibited some differentiation.

Day 3 control cultures were seeded on the same day as the parallel bioreactor cultures into $2 \mathrm{D}$ culture flasks or multiwell plates coated with gelatine, but without MEF preseeding. Thus, they contained only those feeder cells that had been transferred together with the mESC from the previous passage, analogous to the bioreactor cultures.

\section{Culture Media}

Cells were cultured with DMEM containing 1,000 U/ml leukemia inhibitory factor (Esgro), 15\% FCS, penicillin-streptomycin, L-glutamine, $\beta$-mercaptoethanol, nonessential amino acids and nucleosides. All media components were purchased at Biochrom, except for leukemia inhibitory factor, FCS, $\beta$-mercaptoethanol and nonessential amino acids and nucleosides, which were purchased from Chemicon International (Calif., USA).

\section{Culture of Cells in the Bioreactors}

After a conditioning phase of $24 \mathrm{~h}$ with recirculating culture medium, mESC/MEF cocultures from passage 17 (8-ml bioreactor) or passage $18(800-\mathrm{ml}$ bioreactor) were inoculated into the bioreactors without additional MEF cells. The cell compartment was supplied with $100 \mathrm{ml} / \mathrm{min}(8-\mathrm{ml}$ bioreactor) or $600 \mathrm{ml} / \mathrm{min}$ (800-ml bioreactor) of a gas mixture consisting of $95 \%$ air and $5 \%$ $\mathrm{CO}_{2}$. Bioreactors were perfused at a constant recirculation rate of $40 \mathrm{ml} / \mathrm{min}$ (8-ml bioreactor) or $250 \mathrm{ml} / \mathrm{min}$ ( $800-\mathrm{ml}$ bioreactor). Fresh medium was supplied with an increasing feed rate from 6 to $12 \mathrm{ml} / \mathrm{h}(8-\mathrm{ml}$ bioreactor) or from 40 to $160 \mathrm{ml} / \mathrm{h}(800-\mathrm{ml}$ bioreactor). The bioreactors were maintained at $37^{\circ} \mathrm{C}$. Daily mea- surements of $\mathrm{pH}, \mathrm{pO}_{2}, \mathrm{pCO}_{2}$ and buffer capacity were used to adjust medium perfusion and gas supply rates (I-stat, Abbott, East Windsor, N.J., USA; ABL 5, Radiometer Copenhagen, Copenhagen, Denmark).

\section{Metabolic Parameters}

Samples from culture perfusates were taken on a daily basis for biochemical analyses. The metabolic activity of the cells was determined by measuring glucose consumption and lactate production, while potential cell damage was detected by cellular lactate dehydrogenase release. Analyses were performed with assays adapted for an automated clinical analyzer (Roche/Hitachi Modular, Roche Diagnostics GmbH, Germany) according to the manufacturer's instructions.

\section{Bioreactor Cell Sample Harvest}

At the end of the scheduled culture periods, the bioreactors were shut down and the tubes were disconnected. The upper bioreactor lid was opened and the cell mass including the capillary layers was cut from the surrounding potting and transferred into a sterile glass vessel. The contents of the $800-\mathrm{ml}$ bioreactor were divided into 4 sections for cell counting, as described below, teratoma studies, FACS analysis and histology/immunofluorescence, respectively.

\section{Histology and Immunofluorescence}

Material from different locations within the bioreactor cell compartment was fixed in $4 \%$ paraformaldehyde solution prior to paraffin embedding and sectioning.

Antibodies and Dilutions. Primary antibodies: mouse anti- $\alpha-$ smooth muscle actin (cross-reactivity with human, 1:500; CBL171, Chemicon, Billerica, Mass., USA); rabbit antimammalian neuronal class III $\beta$-tubulin (1:250; PRB-435-P, Biosite, Princetown, N.J., USA); goat anti-mouse FoxA2 (HNF3 $\beta$, cross-reactivity with human, 1:200; Sc-6554, Santa Cruz, Delaware, Calif., USA); mouse anti-rat nestin (cross-reactivity with human, 1:200; 611658, BD Transduction laboratories, San José, Calif., USA); mouse antihuman Oct-4 (1:250; Sc-5279, Santa Cruz); mouse anti-mouse stage-specific embryonic antigen-1 (SSEA-1; cross-reactivity with human; MC-480, Developmental Studies Hybridoma Bank, University of Iowa, Iowa, USA). Secondary antibodies: goat $\alpha$-mouse IgG2a-TRITC (1:500; 1080-03, Southern Biotech, Los Angeles, Calif., USA); donkey $\alpha$-goat IgG-Cy3 (1:500; 705-165-147, Jackson Immunoresearch, West Grove, Pa., USA); goat $\alpha$-mouse IgG-Alexa 488 (1:500; A-11029, Molecular Probes, Eugene, Oreg., USA); donkey $\alpha$-rabbit IgG-Alexa 594 (1:500; A-21207, Molecular Probes); goat $\alpha$-mouse IgG1-Cy3 (1:500; 115-165-205, Jackson Immunoresearch); goat anti-mouse IgG-Cy2 (1:1,000; 115-225-003, Dianova).

Staining Procedure. Samples were deparaffinized and rehydrated according to standard procedures using xylene as an organic solvent and a decreasing ethanol gradient to rehydrate the sections. Hematoxylin-eosin (HE) or toluidine blue staining of sections was performed according to standard laboratory procedures. For immunofluorescence, the antigen was retrieved by boiling the sections in $0.01 \mathrm{M}$ citric acid, $\mathrm{pH}$ 6.0. After cooling down the sections at room temperature for about $15 \mathrm{~min}$, the sections were washed twice in phosphate-buffered saline (PBS) and then permeabilized for 5 min in PBS supplemented with $0.5 \%$ Triton X-100 (T8532, Sigma-Aldrich, Germany). Primary antibodies diluted in 
staining buffer (PBS supplemented with $0.1 \%$ Triton X-100) were added and incubated with the sections overnight at $4{ }^{\circ} \mathrm{C}$. Subsequent to threefold washing with $\mathrm{PBS}$, the secondary antibodies diluted in staining buffer were added to the sections and incubated at room temperature for another $2-3 \mathrm{~h}$. Afterwards, the sections were washed 3 times in PBS with the addition of DAPI $(1: 1,000$; D9542, Sigma-Aldrich) in the last washing step. Sections were mounted with the antifading fluorescent mounting medium from Dako Cytomation (s3023). Microphotographs were taken using a Zeiss Axioskop 40 microscope with a Zeiss Axiocam digital camera and Zeiss Axiovision software (Zeiss, Oberkochen, Germany). The microphotographs were enhanced for brightness/contrast using Adobe ${ }^{\circledR}$ Photoshop; color balance was used to enable the visibility of specific stainings breaking through the DAPI staining.

\section{Transmission Electron Microscopy}

For transmission electron microscopy, material from the bioreactor cell compartment was fixed with $5 \%$ glutaraldehyde (Serva, Heidelberg, Germany). After immersion for $30 \mathrm{~min}$ in $60 \mathrm{mM}$ phosphate buffer, $\mathrm{pH} 7.3$, the cellular aggregates were postfixed in $2 \% \mathrm{OsO}_{4}$ (Paesel + Lorei, Frankfurt, Germany) for $2 \mathrm{~h}$, progressively dehydrated in ethanol and then embedded in araldite (Serva). Ultrathin sections were contrasted with uranyl acetate and Reynolds lead citrate (Chroma, Münster, Germany) before electron microscopic examination using a Zeiss 902 (Zeiss).

\section{Capacity of in vivo Teratoma Formation}

The capacity of mESC expanded in the $800-\mathrm{ml}$ bioreactor to form teratomas in vivo was investigated using 8-week-old 129/S6 mice. A total of $1.5 \times 10^{7}$ cells from the cell suspension used for bioreactor inoculation (control) or from the bioreactor after 3 days of mESC expansion were mixed 1:2 (vol:vol) with Matrigel (BD Biosciences) and injected subcutaneously into 5 (control) or 10 (bioreactor) recipient mice. Body weight and tumor size were measured once a week. After 56 days, the mice were sacrificed and tumors were removed for pathohistological analysis. HE staining of teratoma sections showed that the mESC had differentiated into structures typical for of all 3 germ layers, which confirms preservation of the pluripotent character of the mESC during cell expansion in the bioreactor. Structures observed included stratified squamous epithelium, hair follicle-like structures and neural-like tissue (ectoderm), solid growing epithelium islets and glandular epithelium (endoderm), connective tissue, muscle-like tissue, chondral-like tissue and bone-like tissue/bone marrow (mesoderm). The mitosis rate was moderately to strongly increased in epithelial areas.

\section{Quantitative Real-Time PCR}

mRNA analysis was performed using cells from the suspension inoculated into the bioreactor (day 0 ), from the $800-\mathrm{ml}$ bioreactor after 3 days of mESC expansion (day 3 ) and from the 3-day control dish culture. Total RNA was isolated from the samples using the RNeasy Mini Kit (Qiagen, Valencia, Calif., USA) according to the manufacturer's instructions. RNA concentrations were measured with a spectrophotometer, and $500 \mathrm{ng}$ of RNA served as a template to synthesize complementary DNA with the RevertAid ${ }^{\mathrm{TM}} \mathrm{H}$ Minus First Strand cDNA Synthesis Kit (MBI Fermentas, St. Leon-Rot, Germany) with random hexamers. The quality of the cDNA samples was confirmed by ethidium bromide staining in a $3 \%$ agarose gel. Quantitative mRNA expression was conducted with a TaqMan
Gene Expression Assays system (Applied Biosystems, Foster City, Calif., USA), and quantitative real-time (RT)-PCR was performed using an ABI Prism 7700 (Applied Biosystems). Each cDNA template was mixed with PCR master mix and mouse-specific primer sets for Oct-4 (Mm00658129_gH), Nanog (Mm02019550_s1), Sox2 (Mm00488369_s1) and GAPDH (Mm99999915_g1), which were all purchased from Applied Biosystems. Data were analyzed with the ABI Prism 7700 Sequence Detection System software (version 1.1). Expression of specific genes was normalized to that of the housekeeping gene (GAPDH).

\section{Flow Cytometry}

We used FACS to analyze the differentiation state of cells from the inoculated cell suspension (day 0), cells harvested after 2, 3 or 4 days from the 8-ml bioreactor and 2D culture dishes and cells harvested after 3 days from the $800-\mathrm{ml}$ bioreactor. The cell compartment of the bioreactors was gently rinsed by injecting $+4^{\circ} \mathrm{C}$ PBS into the upper port and draining from the lower port. The cells rinsed out were collected and washed by centrifugation. After opening the lid of the bioreactor, some portions of the woven membranes were carefully removed and immersed in $5 \mathrm{ml}$ of $0.05 \%$ trypsin- $0.02 \%$ EDTA solution. Enzymatic incubation was performed for 3 min to obtain a single-cell suspension. The cells obtained by rinsing and those trypsinized were combined and cell number and viability were examined using trypan blue exclusion. One million cells were used for each antibody staining. Specific antibodies and appropriate isotype controls used in this assay were the following: FITC-conjugated rat anti-Oct-4 IgG2b (Cat. No. IC1759F, R\&D Systems), FITCconjugated rat IgG2b isotype (Cat. No. IC013F, R\&D Systems), phycoerythrin-conjugated anti-SSEA-1 mouse IgM (1:250; Cat. No. FAB2155P, R\&D Systems) and phycoerythrin-conjugated mouse IgM isotype control (Cat. No. 12-4752, eBioscience). FACS data were acquired on FACScalibur and FACScalibur 2 and analyzed by Cell Quest Pro software (BD Biosciences, Franklin Lakes, N.J., USA).

\section{Determination of the Total Cell Number in the 800-ml}

Bioreactor after Expansion

To address the practical problem of counting the entire cell number from the 800 - $\mathrm{ml}$ bioreactor, we estimated the resulting cell yield by comparing biological results considering weight-defined bioreactor cell compartment volumes (6 samples from different cell compartment locations) with the results of known cell numbers from 2D standard culture dishes. Using dishes with 4 different mESC culture densities with 3 repeats, we correlated daily culture glucose consumption rates, as well as end-point protein and DNA data with cell chamber counting results for cell numbers after cell trypsinization and single-cell dissociation. After cell disruption, protein and DNA were purified for Coomassie assay/semiquantitative gel electrophoresis and DNA quantification, respectively.

Coomassie Plus ${ }^{T M}$ Protein Assay. Defined numbers of mESC from $2 \mathrm{D}$ culture $\left(2.3 \times 10^{6}, 5.6 \times 10^{6}, 1.2 \times 10^{7}, 2.04 \times 10^{7}\right)$ or weight/volume-defined cells from bioreactor samples were homogenized in a $10 \%$ sucrose solution supplemented with $0.04 \%$ triethanolamine and proteinase inhibitor (Complete ${ }^{\mathrm{TM}}$ Protease inhibitor cocktail tablets, Roche Diagnostics) buffer (1 ml per sample). Samples were further treated with 3 cycles of ultrasound (5 s each per cycle) and centrifuged at $1,000 \mathrm{~g}$ for $15 \mathrm{~min}$ at $4^{\circ} \mathrm{C}$ to 
remove the nuclei. Protein concentrations were measured in the samples according to the instructions of the manufacturer using albumin as a standard. Coomassie Plus was obtained from Pierce Biotechnology (Bonn, Germany).

Semiquantitative Gel Electrophoresis. Samples were homogenized as described above for the Coomassie Plus assay. The supernatants were mixed with heat sample buffer [50 $\mathrm{ml}$ of tris $(2 \mathrm{M})$ SDS, pH 6.8: $4 \mathrm{~g}$ of SDS, $20 \mathrm{ml}$ of glycerin, $10 \mathrm{ml}$ of mercaptoethanol, $600 \mu \mathrm{l}$ of $1 \%$ bromophenol blue] and denatured at $95^{\circ} \mathrm{C}$ for $5 \mathrm{~min}$. Then, $50-\mu \mathrm{l}$ samples from mESC suspensions of defined cell concentrations or from bioreactor cell material were separated by polyacrylamide gel electrophoresis using $8-10 \%$ acrylamide minigels. Gels were stained in Coomassie Plus solution, scanned and analyzed with spot densitometric analysis software (ImageQuant 5.2) to determine the correlation between density and cell number.

DNA Quantification. DNA from samples was purified using the Puregene Tissue Core Kit B (Qiagen). Using a spectrophotometer (ND-1000, Nanodrop, Wilmington, Del., USA), the quantity of DNA contained in defined numbers of mESC $\left(4 \times 10^{5}, 8 \times\right.$ $10^{5}, 1.6 \times 10^{6}$ ) was measured. The purity of the samples was confirmed by the wavelength profile.

Glucose Consumption. Glucose consumption was measured as described above under Metabolic Parameters.

Bioreactor Cell Count Estimation. Excel (version 11.3.5, Microsoft, Redmont Wash., USA) was used to confirm linear function ranges of biologic data and to calculate cell numbers in the bioreactor samples by comparing biologic data from unknown cell numbers in bioreactors to biologic data from well-defined cell numbers in culture dishes.

\section{Results}

The 4-compartment design of the bioreactor and its compartments are described in the legend to figure 1. To operate the bioreactors, a processor-controlled perfusion and gas supply device was developed with modular pump units for various bioreactor scales (fig. 1D).

We cultured 129/SVEV mESC (passage 17) with mitomycin-inactivated MEF strain CD-1 [Smith, 2001] and leukemia inhibitory factor [Williams et al., 1988] in conventional $2 \mathrm{D}$ and $8-\mathrm{ml}$ cell compartment $3 \mathrm{D}$ systems over 2, 3 or 4 days, to compare cell growth and to define the time window for attaining maximal expansion before cells enter stages of enhanced differentiation. Each bioreactor was inoculated with $2.5 \times 10^{7} \mathrm{mESC}$ and approximately $1.5 \times 10^{7} \mathrm{MEF}$ from the previous passage (without further MEF addition).

Examination of bioreactor cultures showed cells aggregated between the capillaries, resulting in a visible, high-density cell mass after 3 or more days of culture (fig. 2A). FACS analysis of the inoculated cells (day 0) or cells harvested from the bioreactors (2, 3 and 4 days) showed that $83,81,80$ and $63 \%$ (corresponding to $0,2,3$ and 4 days) of the cells were positive for Oct- 4 [Solter and Knowles, 1978] and 98, 97, 98 and 83\% were positive for SSEA-1 [Nichols et al., 1998]. Oct-4 and SSEA-1 immunoreactivity staining showed similar marker expression and distribution on days 2 and 3 in 2D and 3D cultures, while culture times longer than 3 days resulted in an increase in differentiation markers in both groups (fig. 3). Quantitative RT-PCR confirmed these data, showing comparable expression of Oct-4, Nanog and Sox 2 genes in $2 \mathrm{D}$ and $3 \mathrm{D}$ cultures.

To demonstrate technology scale-up, we cultured mESC (passage 18) in one 800-ml cell compartment prototype using experimental conditions comparable to those in the 8-ml bioreactors and proportionally increased medium and gas flow rates. The total inoculated cell number was $1.29 \times 10^{9}$, and the cell suspension con-

\section{(For figures see next page.)}

Fig. 1. Bioreactor technology. A Smallest repetitive capillary membrane unit with compartments for medium perfusion (II, IV), oxygenation (III) and cells (I). The cells are not shown; the dots represent mass exchange distribution. B Single units forming capillary bundles. C 3D arrangement of capillaries within the cell compartment. The cells are not shown between the capillaries (for cell position see fig. 2A). Compartments can be perfused independently as shown in $\mathbf{B}$, addressing the reduction of gradient distances between capillary units and enhancing mass exchange. All membrane compartments are interwoven, forming a tight network (C). The capillaries of each compartment are bundled towards the inlet and outlet heads and connected to tube systems for perfusion (B). Cells are inoculated via 16 (8-ml prototype) or 48 (800-ml prototype) open-ended tubes, allowing cell distribution within the cell compartment. Interweaving the smallest capillary units (A) allows scale-up without changing the smallest units, as shown in C. D Processor-controlled perfusion device with a modular multichannel pump unit with medium perfusion and substitution pumps for one 800-ml bioreactor or up to four 8 -ml bioreactors and an $\mathrm{N}_{2} / \mathrm{CO}_{2} / \mathrm{O}_{2} /$ air gas supply unit. Bioreactors are kept at a constant temperature under the transparent $37^{\circ} \mathrm{C}$ hood (upper part); monitoring of flow, pressure, temperature and gas $\left(\mathrm{N}_{2} / \mathrm{CO}_{2} / \mathrm{O}_{2} /\right.$ air $)$ within the perfusion circuit is provided. To demonstrate their size, we placed an additional $800-\mathrm{ml}$ bioreactor (left) and 8-ml bioreactor (right) at the front.

Fig. 2. A One layer of the capillary membrane network from an 8-ml bioreactor before cell inoculation (upper left) and after 6 days of culture (lower right); aggregates are visible between the fibers. B Toluidine blue staining of cells obtained from an 800-ml bioreactor after day 3. Inset: SSEA-1 immunoreactivity (green) on day 3 in the $800-\mathrm{ml}$ bioreactor culture (blue: DAPI staining of cell nuclei). C Glucose consumption and lactate production in the 800 - and 8-ml bioreactors versus glucose consumption in $2 \mathrm{D}$ control dishes over 3 days. Values are given as consumption or production rates per hour per bioreactor/culture flask. Thus, the extremely different scales of the bioreactors and cell numbers are reflected by these medium parameters. 
1

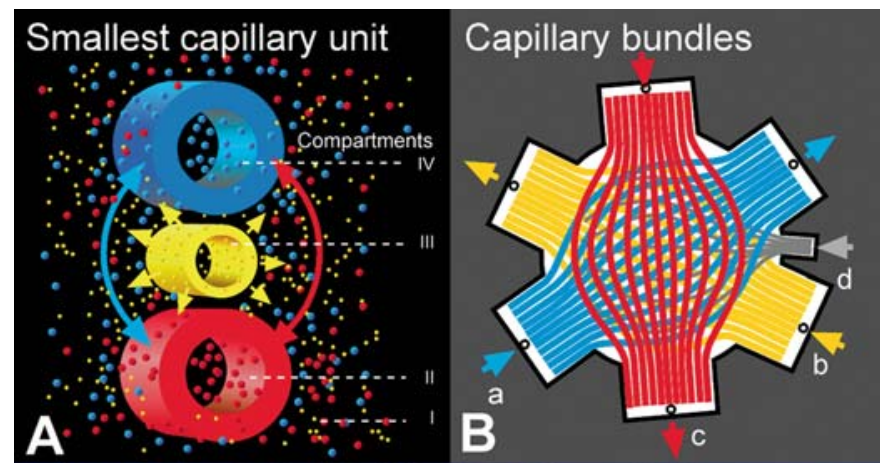

Interwoven capillary bundles

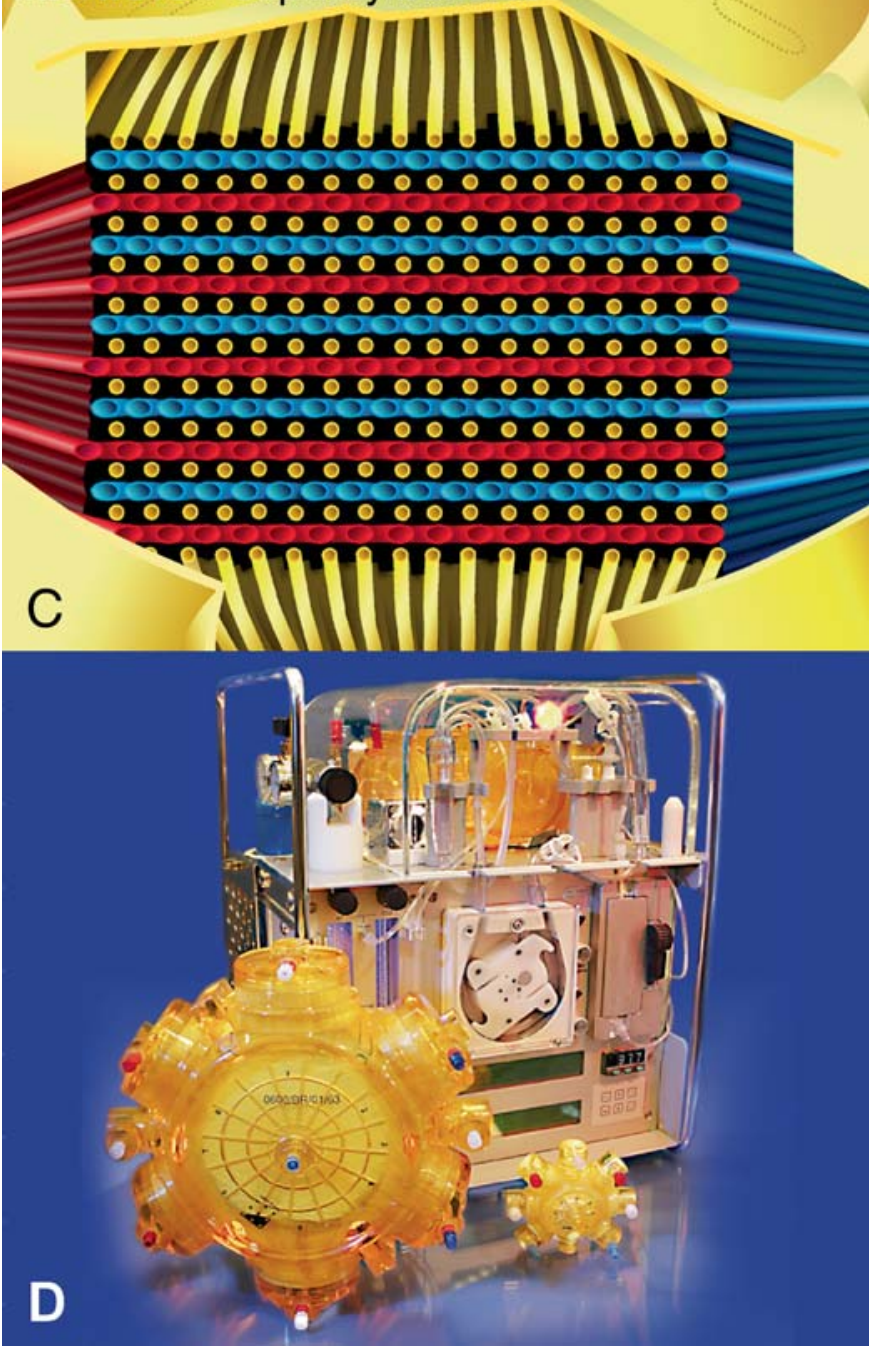

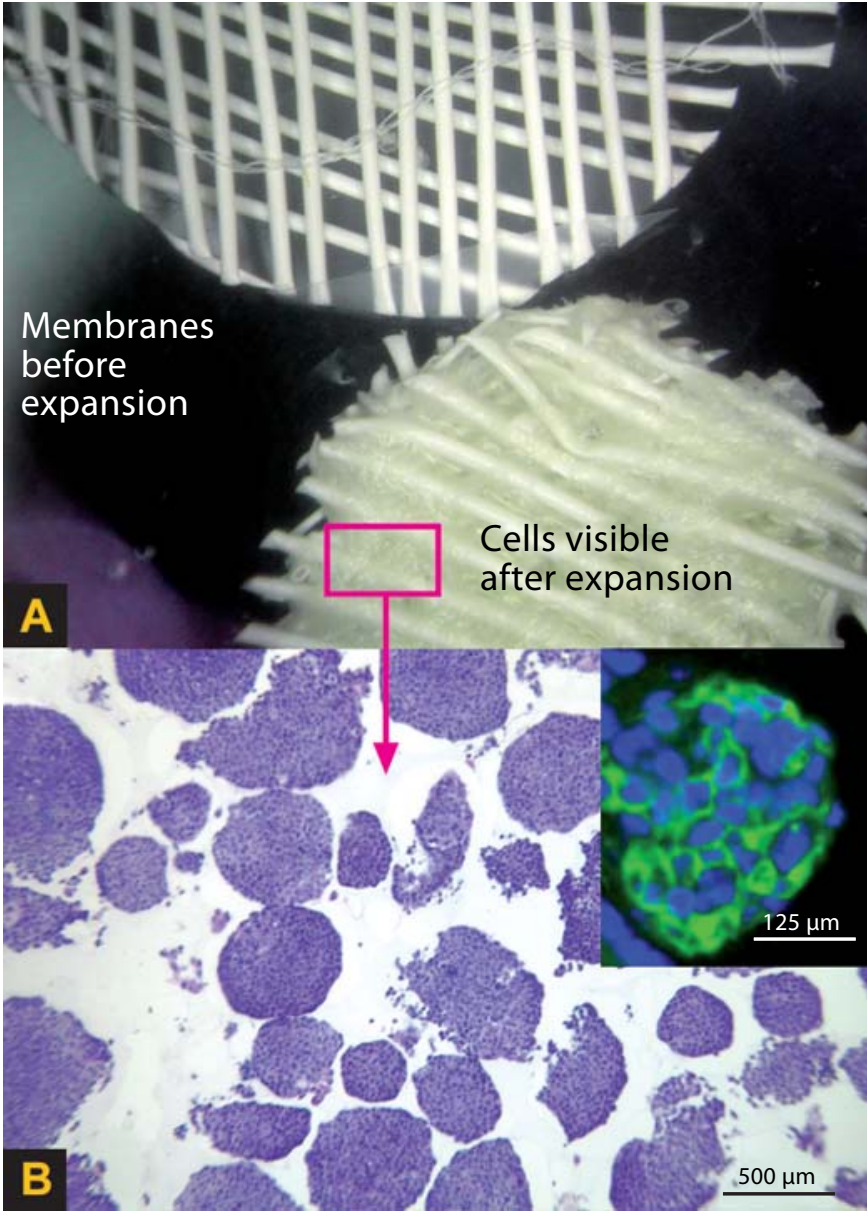

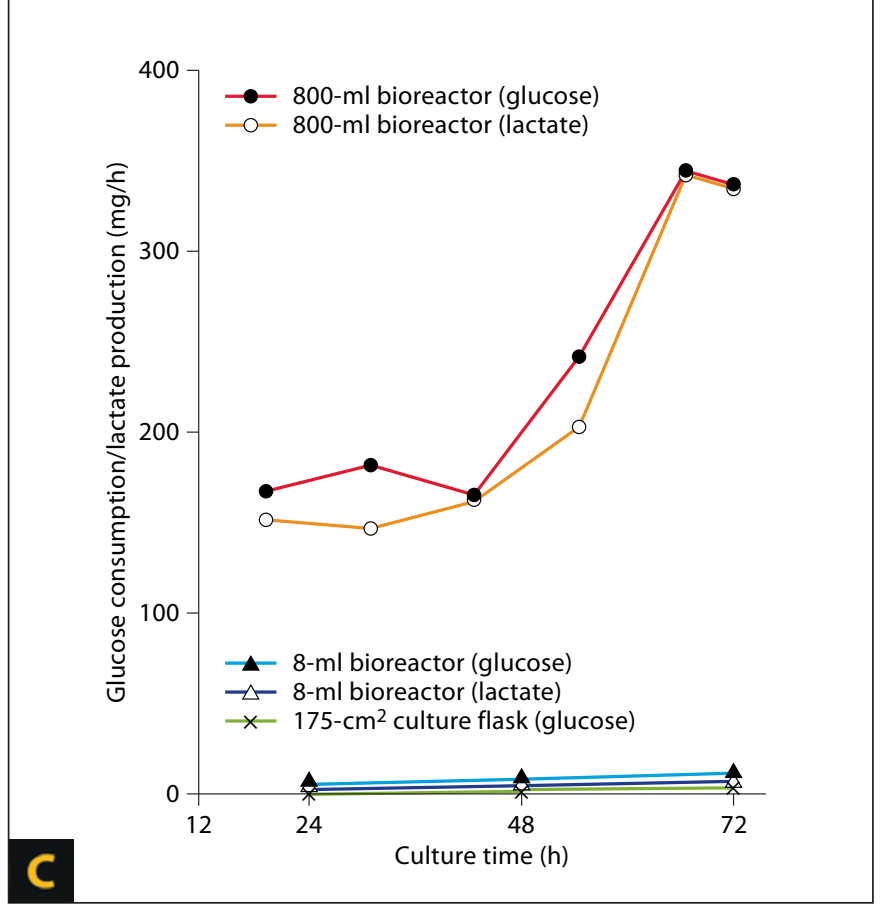



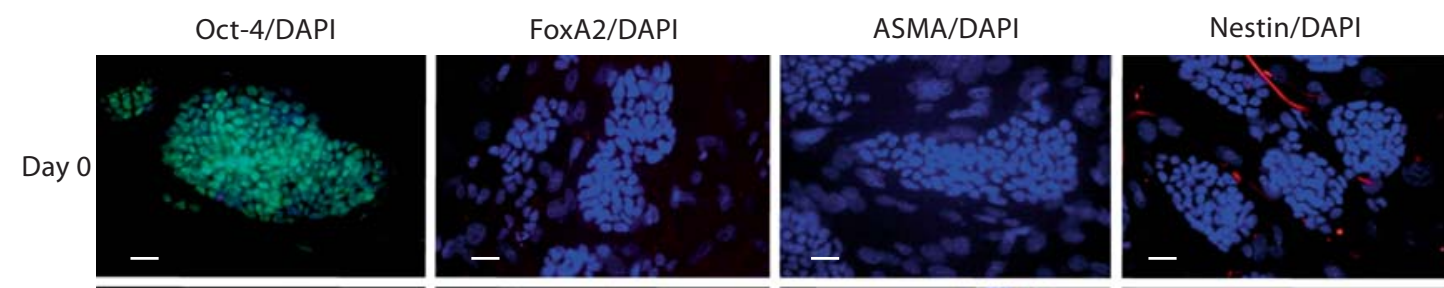

Class III $\beta$-tubulin/DAPI
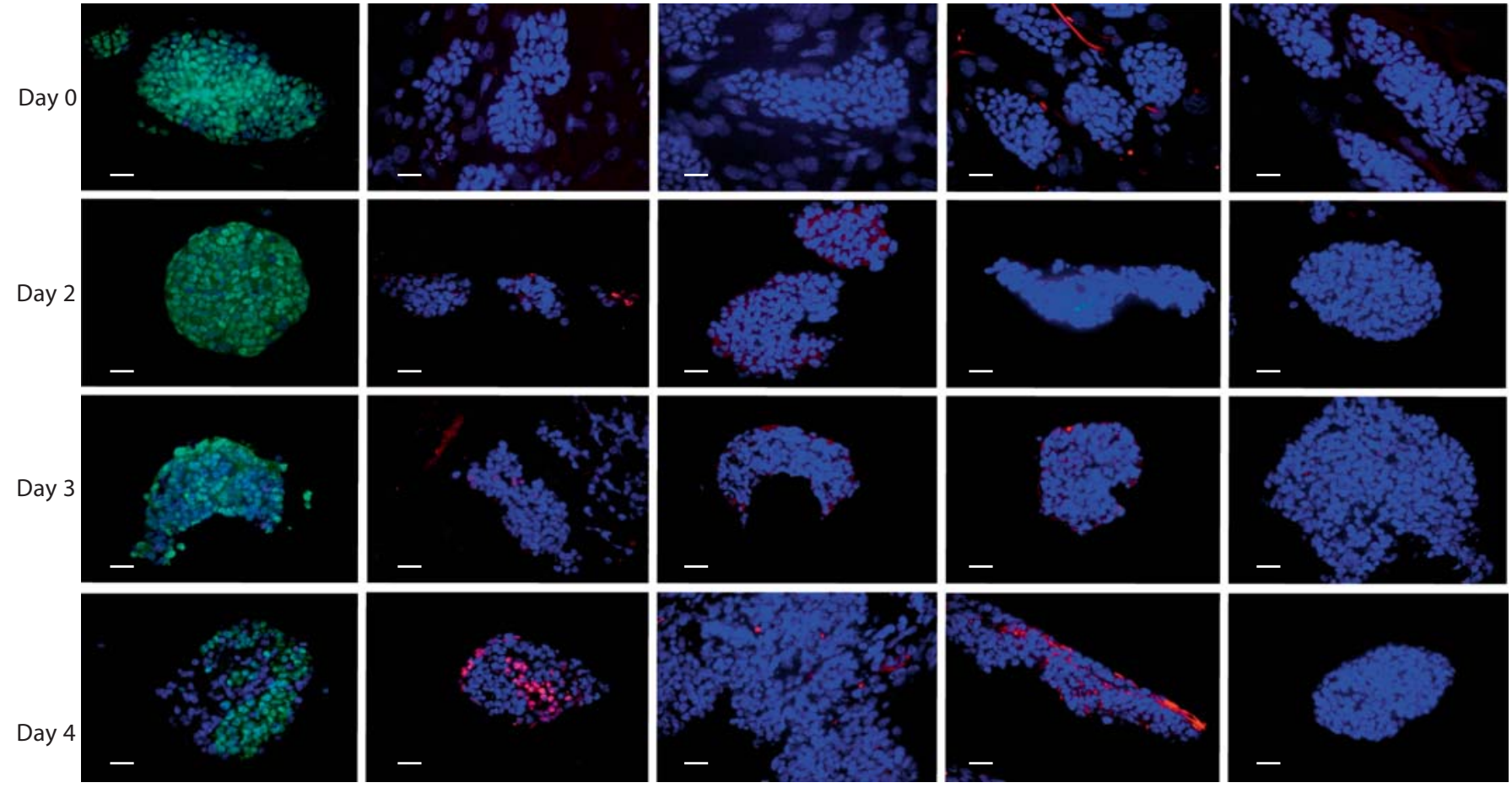

Fig. 3. Immunoreactivity and DAPI nuclei staining (blue) of 2D mESC cultures before inoculation into the bioreactors (day 0 ) or of cell aggregates after culture in 8-ml bioreactors over 2, 3 or 4 days. Each time point represents an independent culture. Similar marker expression and distribution are seen in $2 \mathrm{D}$ and early $3 \mathrm{D}$ cultures. In prolonged 3D culture, the number of stem cell marker Oct-4-positive cells decreased and cell differentiation immunoreactivity markers increased. Early differentiation towards the
3 different germ layer lineages, indicated by immunoreactivity detection of the transcription factor FoxA2 (endoderm) and intermediate filament nestin (early ectoderm) increased in 4-day bioreactor cultures. Class III $\beta$-tubulin (Tuj-1; late ectoderm, postmitotic neurons) and anti- $\alpha$-smooth muscle actin (ASMA; mesoderm) immunoreactivity staining increased in bioreactor cultures maintained for more than 4 days (not shown). Scale bars $=25 \mu \mathrm{m}$. tained approximately $10 \%$ inactivated MEF from the passage prior to cell inoculation. Based on the described time course results from the 8 -ml bioreactors, we chose a culture time of 3 days.

Glucose consumption and lactate production indicated continuous cell growth in the $800-\mathrm{ml}$ bioreactor (fig. 2C). FACS analysis on days 0 and 3 showed that 96 and $85 \%$ of the cells, respectively, were positive for Oct-4, and 68 and 62\%, respectively, were positive for SSEA-1. Quantitative RT-PCR of the pluripotency marker genes Oct- 4 , Nanog and Sox 2 confirmed comparable differentiation status after 3 days in the 8 - and 800 -ml bioreactors.

Histological and immunoreactivity studies revealed cell aggregates with an average diameter of $530 \pm 146$ $\mu \mathrm{m}$ (fig. 2B). Oct- 4 expression was observed in almost all cells in smaller aggregates. A lack of Oct- 4 expression in the central regions of some larger aggregates indicated central necrosis (fig. 4B), which was confirmed with transmission electron microscopy (fig. 4D, E); this may be avoided by changing the membrane weaving patterns and thus reducing the interfiber distances to limit the space for ESC aggregate growth.

The ability of bioreactor-expanded cells to form teratomas indicates preservation of pluripotency. Cells harvested from the $800-\mathrm{ml}$ bioreactor after 3 days of culture were able to form teratomas in vivo within 56 days after subcutaneous injection into 129/S6 mice. The tumors exhibited the formation of tissue structures belonging histologically to all 3 germ layers (fig. 5).

The resulting cell yield was estimated by comparing analyses from the $800-\mathrm{ml}$ bioreactor to analyses and cell counts from standard culture. Using various methods, the calculated cell yield was $4.68 \times 10^{9}$ cells comparing glucose consumption, $7.72 \times 10^{9}$ cells comparing total protein, $5.37 \times 10^{9}$ cells comparing semiquantitative 
Fig. 4. Immunoreactive coexpression of stem cell markers $(\mathbf{A})$, toluidine blue staining (B) and transmission electron microscopy (C, D) of cells cultured over 3 days in an 800 -ml bioreactor. Cells formed mainly aggregates with a size of $530 \pm 146 \mu \mathrm{m}$ in diameter, corresponding to approximately 4,000 cells. SSEA-1 immunoreactivity was regularly distributed and showed partial coexpression with Oct-4, observed in the vast majority of aggregates (A). Transmission electron microscopy studies (C) showed that aggregates were partly lined by feeder cells (F) characterized by flat shapes and cytoplasmic extrusions, and regularly contained mitotic cells (M). Whereas the majority of aggregates showed an intact cell morphology and ultrastructure, a few aggregates of larger size showed central necrosis $(\mathrm{N})$ as assessed by $\mathrm{HE}$ staining (B) and transmission electron microscopy (D). The necrotic areas lacked Oct- 4 staining (B, inset). This necrosis may be addressed by changing the membrane weaving patterns and thus reducing the interfiber distances to mechanically limit the available space for ESC aggregate growth.
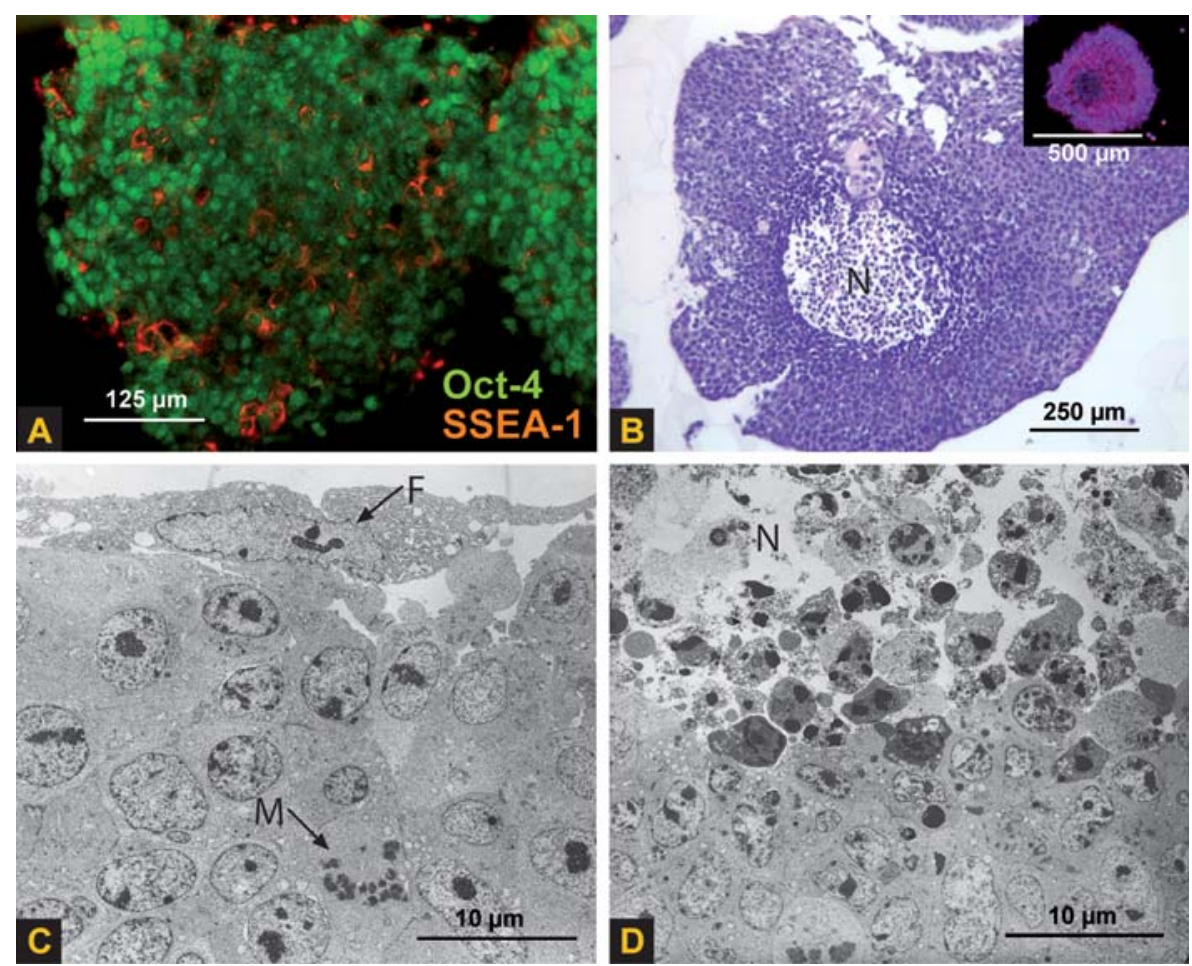
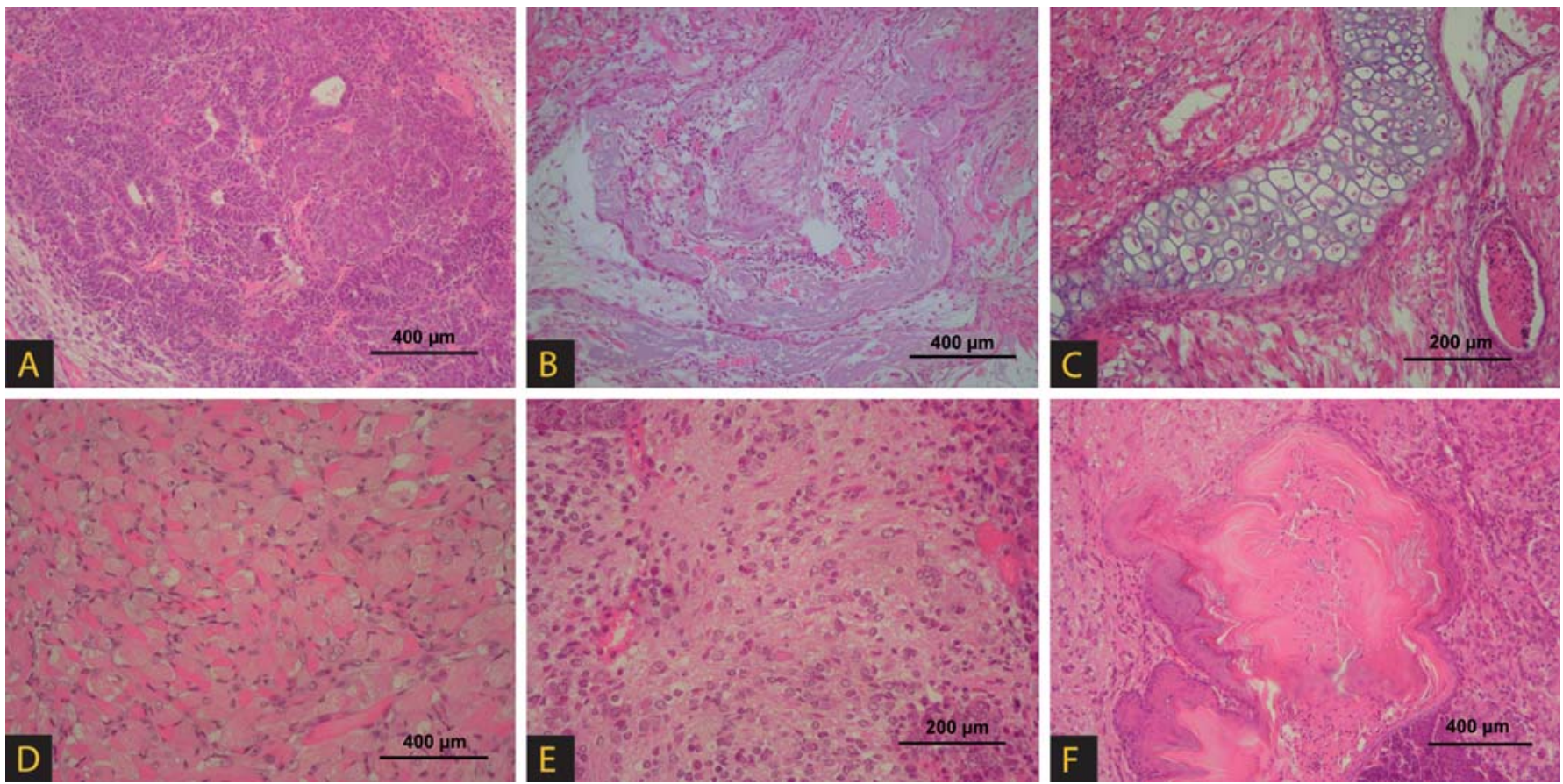

Fig. 5. Cells harvested from the $800-\mathrm{ml}$ bioreactor after 3 days of culture were able to form teratomas in vivo within 56 days after subcutaneous injection into 129/S6 mice. Tumors exhibited the formation of tissue structures belonging histologically to all 3 germ layers, including epithelium-like (A), bone-like (B), chondral-like (C), muscle-like (D), neural-like (E) and cornified squamous epithelium-like (F) structures. 
protein gel electrophoresis and $4.89 \times 10^{9}$ cells comparing DNA quantification, while the cell viability was $82 \%$ (trypan blue exclusion). This indicates a cell amplification factor in the range of 3.6-6.0 within 3 days, comparable to control $2 \mathrm{D}$ Petri dish results $(6.3 \pm 2.8 ; \mathrm{n}=10)$ but on a larger scale.

\section{Discussion}

For the development and implementation of stem cellbased applications in regenerative medicine and applied research, large numbers of cells with well-defined characteristics are needed. Commonly employed ESC culture and differentiation methods utilize $2 \mathrm{D}$ plastic dish surfaces. These represent static open systems with discontinuous medium exchange, which results in periodical changes to the culture environment in the form of accumulation of metabolites and reduction of nutrients in the culture medium between medium changes. They are also labor intensive and prone to error, because they require extensive manual intervention and therefore make handling of larger cell numbers impractical. One approach to standardize ESC production is the automation of certain dish culture steps, including automated T flask culture platforms [Terstegge et al., 2007].

The use of dynamic 3D perfusion bioreactors represents an alternative to dish cultures, since they can provide a more homogeneous environment, make it possible to monitor and control culture parameters and allow easier scale-up. It has been shown that medium perfusion is beneficial even in 2D cultures [Fong et al., 2005]. Perfusion facilitates continuous nutrient and oxygen supply [Ouyang and Yang, 2008] in larger cell masses. The reduction of metabolite accumulation, provision of a continuous supply of nutrients and control of parameters such as oxygen partial pressure and $\mathrm{pH}$ all result in the maintenance of favorable growth conditions.

The types of bioreactors described for ESC culture and differentiation include stirred bioreactors (e.g. Spinner flasks), rotary systems such as slow-turning lateral vessels [Come et al., 2008; Hwang et al., 2009] or fibrousbed bioreactors [Li et al., 2003; Ouyang and Yang, 2008]. Because ESC become adherent in colonies and are dependent on tight cell-cell contacts, they do not grow as single cells in suspension; to culture the cells in stirredtank bioreactors, they are either maintained as aggregates or adhered to microcarriers. Undifferentiated expansion of mouse ES cells has been shown using microcarriers [Abranches et al., 2007; Fernandes et al., 2007] as well as in carrier-free suspension [zur Nieden et al., 2007]. The culture of ESC as aggregates in suspension is limited by the size of the cell clusters. If the clusters become too large in size, mass transport into their center is reduced, e.g. transport of oxygen and nutrients, leading to cell necrosis. The use of microcarriers reduces the shear stress on the cells, but only low cell expansion and seeding efficiencies could be achieved [Phillips et al., 2008; Nie et al., 2009]. The use of microcarriers is also limited by their available surface area, thereby requiring frequent cell dissociation and passaging, which leads to cell loss due to low seeding efficiencies and the fragility of the cells. To solve these problems, novel techniques are needed.

In the present study, we demonstrated the cultivation of a cell mass of $5 \times 10^{9} \mathrm{mESC}$. This would require approximately 800 Petri dishes $(100 \mathrm{~mm})$ or $280 \mathrm{~T}-175$ flasks and 14 person hours daily, while the bioreactor routine required 1 person hour daily. The outer volume of $280 \mathrm{~T}$ 175 flasks represents 252 liters, requiring $4-5$ incubators and corresponding sterile workbench space. This compares to a 5.2-liter outer volume of the bioreactor, requiring 1 perfusion system as shown in figure 1D.

As a functional assay to demonstrate the usefulness of the resulting cells, we tested the cells in a mouse model. Formation of teratoma (benign) or teratocarcinoma (malign), spontaneously occurring germ cell tumors that contain somatic tissues derived from each of the 3 embryonic germ layers [Blum and Benvenisty, 2008], by ESC in immunodeficient mice models is the most widely accepted test for the developmental potential of isolated and cultured ESC [Heins et al., 2004; Valbuena et al., 2006; Adewumi et al., 2007]. Studying our expanded ESC in teratomas showed that they can develop into a highly organized structure consisting of ordered arrangements of different tissue types that in some ways replicate organogenesis within the embryo [Przyborski, 2005; Aleckovic and Simón, 2008].

The features of a culture system should allow for the expansion of undifferentiated ESC and/or directed reproducible differentiation into mature cell types. With most available systems, expansion can be achieved in the early culture periods, while differentiation cannot be avoided after extended culture times. This suggests that for the development of suitable culture models, associated passaging methods also need to be considered. Besides offering more physiologically dynamic culture conditions with continuous medium exchange and decentralized perfusion and gas supply, the technology presented here enables culture of larger cell numbers in a single sys- 
tem suitable for automated closed-system cell production. After enzymatic passaging within the bioreactor and flushing of the contents, a transfer into one largescale or several small-scale bioreactors is possible via flow heads on opposite sides of the cell compartment.

Alternatively, since the technology allows variation and control of system parameters (including medium/ factor supply pattern, perfusion rates, oxygen tensions), the bioreactor with expanded cells may also be used for follow-up studies on ESC differentiation, by applying specific differentiation protocols following expansion in the same closed system. However, such possibilities would need to be investigated in further studies.

\section{Acknowledgements}

This study was partially supported by grants from the National Institutes of Health (NIH R21 EB005739-01, J.C.G.), the European Commission (EU STREP-CT-2005-018940, J.C.G.) and the Federal Ministry of Education and Research, Germany (01GN0526 and 01GG0731/0313911, K.Z.). We are grateful for the discussions, excellent help and assistance with mESC cultures from Dr. Chris Navara (Pittsburgh Development Center), transmission electron microscopy from Petra Schrade (Department of Anatomy, Charité Berlin, Germany), histopathological analysis of teratomas from Dr. W. Haider (Institute for Animal Pathology, Berlin, Germany), graphics from Wolfgang Mudra (Charité) and photographs from Dan McKeel (McGowan Institute).

\section{References}

Abbott, A. (2003) Cell culture: biology's new dimension. Nature 424: 870-872.

Abranches, E., E. Bekman, D. Henrique, J.M.S. Cabral (2007) Expansion of mouse embryonic stem cells on microcarriers. Biotechnol Bioeng 96: 1211-1221.

Adewumi, O., B. Aflatoonian, L. Ahrlund-Richter, A. Amit, P.W. Andrews, G. Beighton, P.A. Bello, N. Benvenisty, L.S. Berry, S. Bevan, B. Blum, J. Brooking, K.G. Chen, A.B.H. Choo, G.A. Churchill, M. Corbel, I. Damjanov, J.S. Draper, P. Dvorak, K. Emanuelsson, R.A. Fleck, A. Ford, K. Gertow, M. Gertsenstein, P.J. Gokhale, R.S. Hamilton, A. Hampl, L.E. Healy, O. Hovatta, J. Hyllner, M.P. Imreh, J. Itskovitz-Eldor, J. Jackson, J.L. Johnson, M. Jones, K. Kee, B.L. King, B.B. Knowles, M. Lako, F. Lebrin, B.S. Mallon, D. Manning, Y. Mayshar, R.D.G. McKay, A.E. Michalska, M. Mikkola, M. Mileikovsky, S.L. Minger, H.D. Moore, C.L. Mummery, A. Nagy, N. Nakatsuji, C.M. O’Brien, S.K.W. Oh, C. Olsson, T. Otonkoski, K.Y. Park, R. Passier, H. Patel, M. Patel, R. Pedersen, M.F. Pera, M.S. Piekarczyk, R.A.P. Pera, B.E. Reubinoff, A.J. Robins, J. Rossant, P. RuggGunn, T.C. Schulz, H. Semb, E.S. Sherrer, H. Siemen, G.N. Stacey, M. Stojkovic, H. Suemori, J. Szatkiewicz, T. Turetsky, T. Tuuri, S. van den Brink, K. Vintersten, S. Vuoristo, D. Ward, T.A. Weaver, L.A. Young, W. Zhang (2007) Characterization of human embryonic stem cell lines by the International Stem Cell Initiative. Nat Biotechnol 25: 803-816.

Aleckovic, M., C. Simón (2008) Is teratoma formation in stem cell research a characterization tool or a window to developmental biology? Reprod Biomed Online 17: 270-280.

Blum, B., N. Benvenisty (2008) The tumorigenicity of human embryonic stem cells. Adv Cancer Res 100: 133-158.
Come, J., X. Nissan, L. Aubry, J. Tournois, M. Girard, A.L. Perrier, M. Peschanski, M. Cailleret (2008) Improvement of culture conditions of human embryoid bodies using a controlled perfused and dialyzed bioreactor system. Tissue Eng Part C Methods 14: 289-298.

Cukierman, E., R. Pankov, K.M. Yamada (2002) Cell interactions with three-dimensional matrices. Curr Opin Cell Biol 14: 633-639.

Evans, M.J., M.H. Kaufman (1981) Establishment in culture of pluripotential cells from mouse embryos. Nature 292: 154-156.

Fernandes, A.M., T.G. Fernandes, M.M. Diogo, C.L. da Silva, D. Henrique, J.M.S. Cabral (2007) Mouse embryonic stem cell expansion in a microcarrier-based stirred culture system. J Biotechnol 132: 227-236.

Fong, W.J., H.L. Tan, A. Choo, S.K. Oh (2005) Perfusion cultures of human embryonic stem cells. Bioprocess Biosyst Eng 27: 381387.

Gerlach, J.C., K. Mutig, I.M. Sauer, P. Schrade, E. Efimova, T. Mieder, G. Naumann, A. Grunwald, G. Pless, A. Mas, S. Bachmann, P. Neuhaus, K. Zeilinger (2003) Use of primary human liver cells originating from discarded grafts in a bioreactor for liver support therapy and the prospects of culturing adult liver stem cells in bioreactors: a morphologic study. Transplantation 76: 781-786.

Heins, N., M.C.O. Englund, C. Sjoblom, U. Dahl, A. Tonning, C. Bergh, A. Lindahl, C. Hanson, H. Semb (2004) Derivation, characterization, and differentiation of human embryonic stem cells. Stem Cells 22: 367-376.

Hwang, Y.S., J. Cho, F. Tay, J.Y.Y. Heng, R. Ho, S.G. Kazarian, D.R. Williams, A.R. Boccaccini, J.M. Polak, A. Mantalaris (2009) The use of murine embryonic stem cells, alginate encapsulation, and rotary microgravity bioreactor in bone tissue engineering. Biomaterials 30: 499-507.
Li, Y., D.A. Kniss, L.C. Lasky, S.T. Yang (2003) Culturing and differentiation of murine embryonic stem cells in a three-dimensional fibrous matrix. Cytotechnology 41: 23-35.

Martin, G.R. (1981) Isolation of a pluripotent cell line from early mouse embryos cultured in medium conditioned by teratocarcinoma stem cells. Proc Natl Acad Sci USA 12: 76347638.

Monga, S.P., M.S. Hout, M.J. Baun, A. Micsenyi, P. Muller, L. Tummalapalli, A.R. Ranade, J.H. Luo, S.C. Strom, J.C. Gerlach (2005) Mouse fetal liver cells in artificial capillary beds in three-dimensional four-compartment bioreactors. Am J Pathol 167: 12791292.

Nichols, J., B. Zevnik, K. Anastassiadis, H. Niwa, D. Klewe-Nebenius, I. Chambers, H. Scholer, A. Smith (1998) Formation of pluripotent stem cells in the mammalian embryo depends on the POU transcription factor Oct 4 . Cell 95: 379-391.

Nie, Y., V. Bergendahl, D.L. Hei, M.J. Jones, S.P. Palecek (2009) Scalable culture and cryopreservation of human embryonic stem cells on microcarriers. Biotechnol Prog 25: 2031.

Ouyang, A., S.T. Yang (2008). A two-stage perfusion fibrous bed bioreactor system for mass production of embryonic stem cells. Expert Opin Biol Ther 7: 895-909.

Phillips, B.W., R. Horne, T.S. Lay, W.L. Rust, T.T Teck, J.M. Crook (2008) Attachment and growth of human embryonic stem cells on microcarriers. J Biotechnol 138: 24-32.

Powell, K. (2005) Stem-cell niches: it's the ecology, stupid! Nature 435: 268-270.

Przyborski, S.A. (2005) Differentiation of human embryonic stem cells after transplantation in immune-deficient mice. Stem Cells 23: $1242-1250$ 
Smith, A.G. (2001) Embryo-derived stem cells: of mice and men. Annu Rev Cell Dev Biol 17: 435-462.

-Solter, D., B.B. Knowles (1978) Monoclonal antibody defining a stage-specific mouse embryonic antigen (SSEA-1). Proc Natl Acad Sci USA 75: 5565-5569.

Terstegge, S., I. Laufenberg, J.R. Pochert, S. Schenk, J. Itskovitz-Eldor, E. Endl, O. Brustle (2007) Automated maintenance of embryonic stem cell cultures. Biotechnol Bioeng 96: 195-201.
Thomson, J.A., J. Itskovitz-Eldor, S.S. Shapiro, M.A. Waknitz, J.J. Swiergiel, V.S. Marshall, J.M. Jones (1998) Embryonic stem cell lines derived from human blastocysts. Science 282: 1145-1147.

Valbuena, D., A. Galán, E. Sánchez, M.E. Poo, E. Gómez, S. Sánchez-Luengo, D.O. Melguizo, A.D. García, V.N. Ruiz, R.N. Moreno, A. Pellicer, C. Simón (2006) Derivation and characterization of three new Spanish human embryonic stem cell lines (VAL -3 -4 -5) on human feeder and in serum-free conditions. Reprod Biomed Online 13: 875-886.

Williams, R.L., D.J. Hilton, S. Pease, T.A. Willson, C.L. Stewart, D.P. Gearing, E.F. Wagner, D. Metcalf, N.A. Nicola, N.M. Gough (1988) Myeloid leukaemia inhibitory factor maintains the developmental potential of embryonic stem cells. Nature 336: 684-687.
Xu, A.S., L.M. Reid (1999) Lineage and biology and liver; in Lanza, R.P., R. Langer, J. Vacanti (eds): Principles of Tissue Engineering, ed 2. San Diego, Academic Press, chapter 41, pp 559-598.

zur Nieden, N.I., J.T. Cormier, D.R. Rancourt, M.S. Kallos (2007) Embryonic stem cells remain highly pluripotent following long term expansion as aggregates in suspension bioreactors. J Biotechnol 129: 421-32. 